Laser-Induced Damage in DKDP Crystals under Simultaneous Exposure to Laser Harmonics

R. A. Negres, P. DeMange, H. B. Radousky, S. G. Demos

November 3, 2005

Boulder Damage Symposium Boulder, CO, United States September 19, 2005 through September 21, 2005 
This document was prepared as an account of work sponsored by an agency of the United States Government. Neither the United States Government nor the University of California nor any of their employees, makes any warranty, express or implied, or assumes any legal liability or responsibility for the accuracy, completeness, or usefulness of any information, apparatus, product, or process disclosed, or represents that its use would not infringe privately owned rights. Reference herein to any specific commercial product, process, or service by trade name, trademark, manufacturer, or otherwise, does not necessarily constitute or imply its endorsement, recommendation, or favoring by the United States Government or the University of California. The views and opinions of authors expressed herein do not necessarily state or reflect those of the United States Government or the University of California, and shall not be used for advertising or product endorsement purposes. 


\title{
Laser-induced damage in DKDP crystals under simultaneous exposure to laser harmonics
}

\author{
R. A. Negres, P. DeMange, H. B. Radousky and S. G. Demos \\ Lawrence Livermore National Laboratory \\ 7000 East Avenue, Livermore, CA 94551, USA
}

\begin{abstract}
While KDP and DKDP crystals remain the only viable solution for frequency conversion in large aperture laser systems in the foreseeable future, our understanding of damage behavior in the presence of multiple colors is very limited. Such conditions exist during normal operation where, for third harmonic generation, $1 \omega, 2 \omega$ and $3 \omega$ components are present with different energy ratios as they propagate inside the crystal. The objective of this work is to shed light into the damage behavior of frequency conversion crystals during operational conditions as well as probe the fundamental mechanisms of damage initiation. We have performed a series of experiments to quantify the damage performance of pristine (unconditioned) DKDP material under simultaneous exposure to $2 \omega$ and $3 \omega$ laser pulses from a 3-ns Nd:YAG laser system as a function of the laser fluences at each frequency. Results show that simultaneous dual wavelength exposure leads to a much larger damage density as compared to the total damage resulting from separate exposure at each wavelength. Furthermore, under such excitation conditions, the damage performance is directly related to and can be predicted from the damage behavior of the crystal at each wavelength separately while the mechanism and type of defects responsible for damage initiation are shown to be the same at both $2 \omega$ and $3 \omega$ excitation.
\end{abstract}

Keywords: Laser-induced damage, KDP and DKDP crystals, nonlinear frequency conversion

\section{INTRODUCTION}

Potassium hydrogen phosphate $\left(\mathrm{KH}_{2} \mathrm{PO}_{4}\right.$ or KDP) and its deuterated analog (DKDP) are currently the only nonlinear materials suitable as Pockels cells and frequency converters in high-power large-aperture laser systems. ${ }^{1,2}$ Damage thresholds in these materials have increased over time, primarily due to purer raw materials and improvement in the polishing and/or growth processes, though localized damage sites still arise from laser intensities far below that necessary for "intrinsic" dielectric breakdown. ${ }^{3}$ The damage initiation mechanisms using nanosecond pulses still largely defy fundamental understanding despite more than four decades of research. ${ }^{4}$ Damage testing shows a variation, even between neighboring sites, in the initiation threshold intensity. This indicates that the cause of damage at relatively low laser fluences is pre-existing defects.

The damage performance of KDP/DKDP crystals under simultaneous exposure to several harmonics of a nanosecond Nd:YAG 1064-nm laser system is of particular interest because it approximates the conditions taking place during frequency conversion. Previous work has focused exclusively on the damage performance at a particular wavelength while practical application of KDP and DKDP involve the presence of multiple wavelengths. ${ }^{3,5}$ Preliminary experiments during frequency conversion have indicated that damage density is a function of the local $1 \omega, 2 \omega$ and $3 \omega$ fluences. ${ }^{6}$ The latter results suggested that the damage density is higher in the presence of multiple wavelengths than that from $3 \omega$ alone and the presence of $1 \omega$ light does not contribute to the enhanced damage effects.

In this work, we performed a detailed investigation into the damage behavior of an optical material under simultaneous $2 \omega$ and $3 \omega$ irradiation in pristine DKDP crystals exposed to various fluence combinations. The experimental results provide i) information on the damage behavior under operationally relevant excitation conditions, ii) the means to predict performance under multi-wavelength irradiation, and iii) insight into the mechanisms governing damage initiation and the interaction of laser light with the absorbing defects responsible for damage.

Send correspondence to R. A. Negres: E-mail: negres2@llnl.gov, Telephone: 1925423 1425, Fax: 19254230909 


\section{EXPERIMENT}

This study has been enabled by the development of a new damage testing system where the bulk damage density (pinpoint density or PPD) is directly measured as a function of exposure laser fluence. The experimental setup along with the system capabilities have been described in detail elsewhere. ${ }^{7}$ Figure 1 shows a schematic top view of the setup. In short, the co-propagating laser beams at $532(2 \omega)$ and $355 \mathrm{~nm}(3 \omega)$ originating from an Nd:YAG laser system are focused inside the crystal sample using a $200-\mathrm{mm}$ cylindrical lens. The $3 \omega$ and $2 \omega$ beams have pulse durations at FWHM of 2.5 and 4 ns and widths at $1 / \mathrm{e}^{2}$ of $60 \mu \mathrm{m}$ and $80 \mu \mathrm{m}( \pm 2 \mu \mathrm{m})$, respectively. A counter-propagating cw He-Ne diagnostic laser beam at $632.8 \mathrm{~nm}$ is focused at the same location in the crystal in order to illuminate any resulting damage pinpoints following exposure to high laser fluences. Scattered light images are recorded orthogonally to the laser propagation direction using a CCD camera coupled to a long-working distance microscope objective lens. From these images, the total number of damage sites is computed in the region of the crystal exposed to peak laser fluence only, corresponding to a volume of $\sim 0.16 \mathrm{~mm}^{3}$ (the width of the $2 \omega$ beam was used as the depth in calculating the volume). A damage profile (PPD vs. laser fluence) is thus obtained from several images of volumes exposed to different fluences. The DKDP samples used in this work were obtained from two conventionally grown crystal boules ${ }^{1,2}$ and were uncoated, diamond-turn polished, and cut to $1 \times 5 \times 5 \mathrm{~cm}^{3}$ in size. One of the samples exhibited a low damage threshold accompanied by high PPD while the second sample had the opposite behavior, with a high damage threshold and lower PPD $(\sim 10 \times)$.
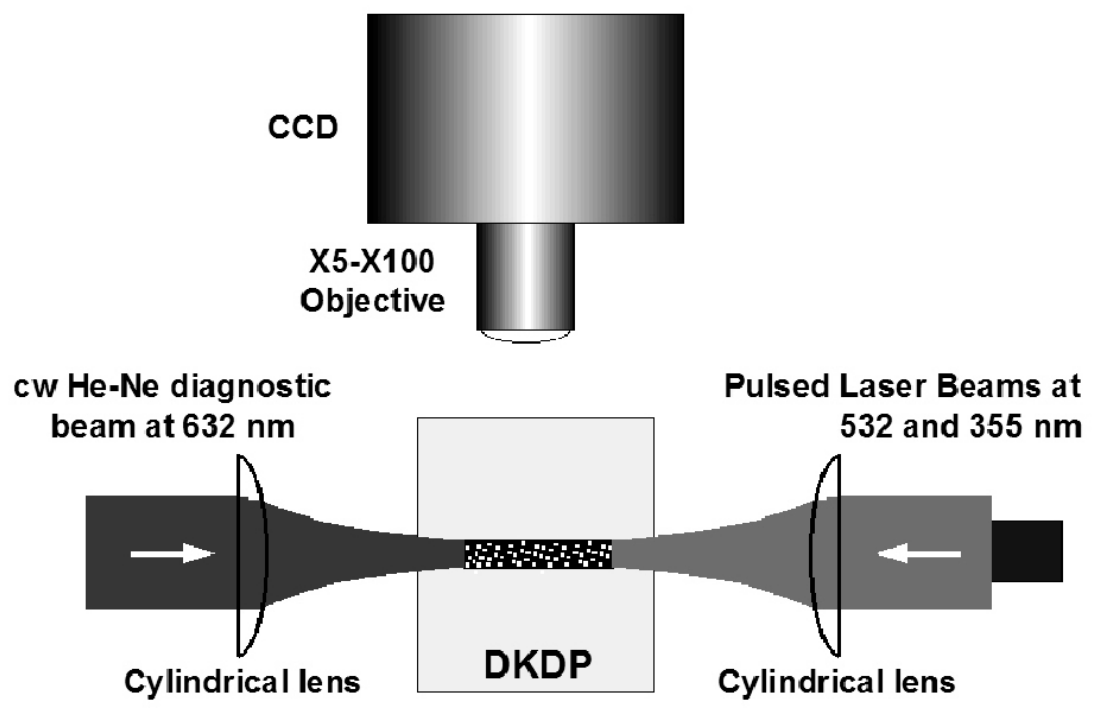

Figure 1. Schematic top view of damage testing system.

\section{RESULTS AND DISCUSSION}

The experimental procedure was the following. Pristine sites in the bulk of our DKDP samples were exposed to either individual pulses at a single wavelength with increasing fluences or to spatially and temporally overlapping $3 \omega$ and $2 \omega$ pulses at various fluence combinations. Figure 2 shows the damage behavior of one of the two samples under simultaneous exposure to combination of fluences at $5,8,12,16,20$, and $24 \mathrm{~J} / \mathrm{cm}^{2}$ at $2 \omega$ and $3,5,8,10,12,15$ and $20 \mathrm{~J} / \mathrm{cm}^{2}$ at $3 \omega$. The data are sorted into sets where the $3 \omega$ fluence is kept constant while the $2 \omega$ fluence is varied [Fig. 2(a)] and vice versa [Fig. 2(b)]. The PPD versus fluence under exposure to each wavelength separately are also shown for comparison and are displayed as profiles at constant fluence of $0 \mathrm{~J} / \mathrm{cm}^{2}$ at the other wavelength in Figs. 2(a) and 2(b). In these experiments, the PPD at each combination of fluences was obtained from the average of at least four tested sites. Similar behaviors were observed in both DKDP samples studied although the PPD at a particular fluence combination varied between each material.

A brief look at the data shown in Fig. 2(a) indicates that the PPD increases nonlinearly with increase in $2 \omega$ fluence and constant fluence exposure at various $3 \omega$ fluences. In contrast, with increase in constant $2 \omega$ fluence, a transition from a nonlinear to a linear increase in the PPD with increase in $3 \omega$ fluence is observed [see Fig. 2(b)].

A first step in our data analysis is to determine the fluences for which the simultaneous presence of the two colors provides the most pronounced damage effects as compared to the expected combined damage from irradiation at each 

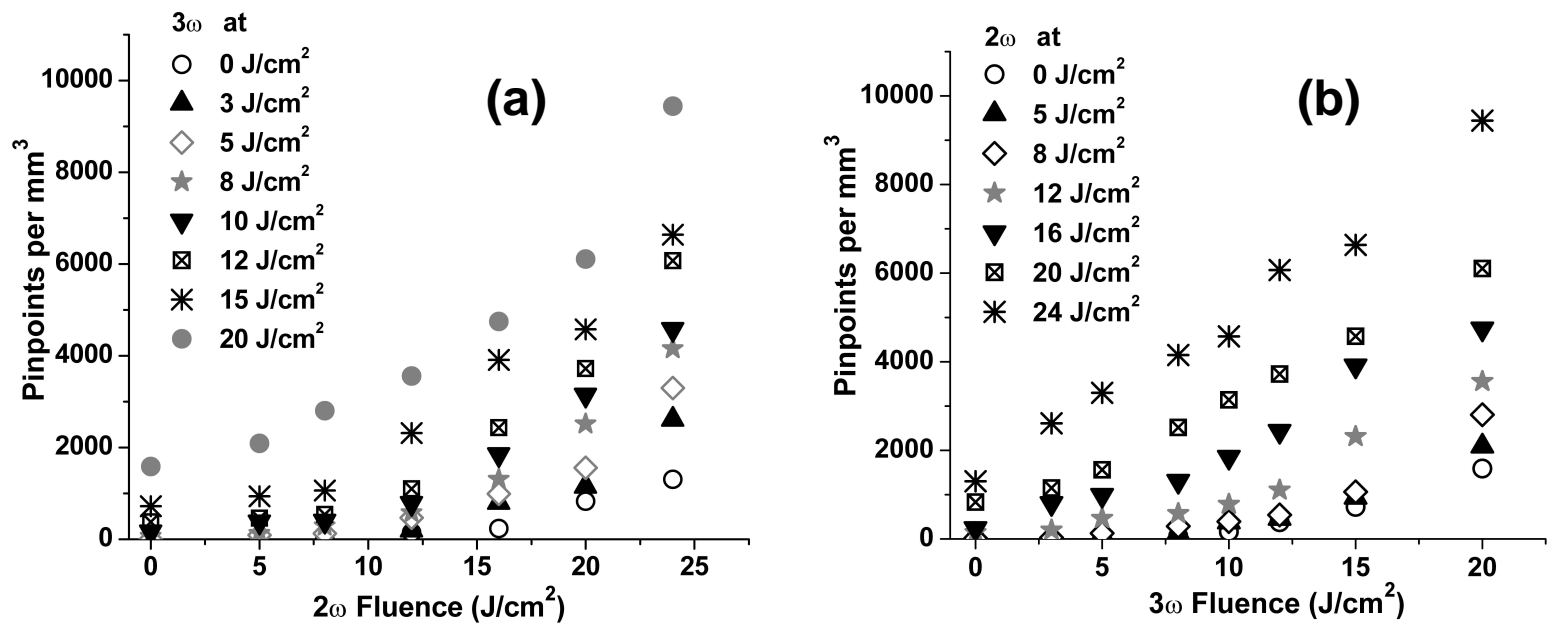

Figure 2. Damage density profiles measured under a matrix of $2 \omega$ and $3 \omega$ fluences: (a) PPD profiles at constant $3 \omega$ fluences versus $2 \omega$ fluence and (b) PPD profiles at constant $2 \omega$ fluences versus $3 \omega$ fluence.

individual wavelength. We thus define the damage amplification factor, $Z$, by the following expression:

$$
Z=\frac{\operatorname{PPD}\left[\Phi_{2 \omega}, \Phi_{3 \omega}\right]}{\operatorname{PPD}\left[\Phi_{2 \omega}\right]+\operatorname{PPD}\left[\Phi_{3 \omega}\right]}
$$

where $\operatorname{PPD}\left[\Phi_{2 \omega}, \Phi_{3 \omega}\right]$ and $\operatorname{PPD}\left[\Phi_{2 \omega(3 \omega)}\right]$ are the PPDs resulting from simultaneous and separate exposure to $2 \omega$ and $3 \omega$ fluences $\left(\Phi_{2 \omega}\right.$ and $\left.\Phi_{3 \omega}\right)$, respectively. Using Eq. 1 and the data presented in Fig. 2, the values of $Z$ can be computed for all $2 \omega$ and $3 \omega$ fluence combinations. Figure 3 illustrates $Z$ profiles versus the $3 \omega$ fluence at constant $2 \omega$ fluences (not all values are shown). We conclude that amplification of damage effects is significant (above unity) when both $2 \omega$ and $3 \omega$ fluences are below $\sim 10-15 \mathrm{~J} / \mathrm{cm}^{2}$, which is the range of fluences where the material is expected to operate.

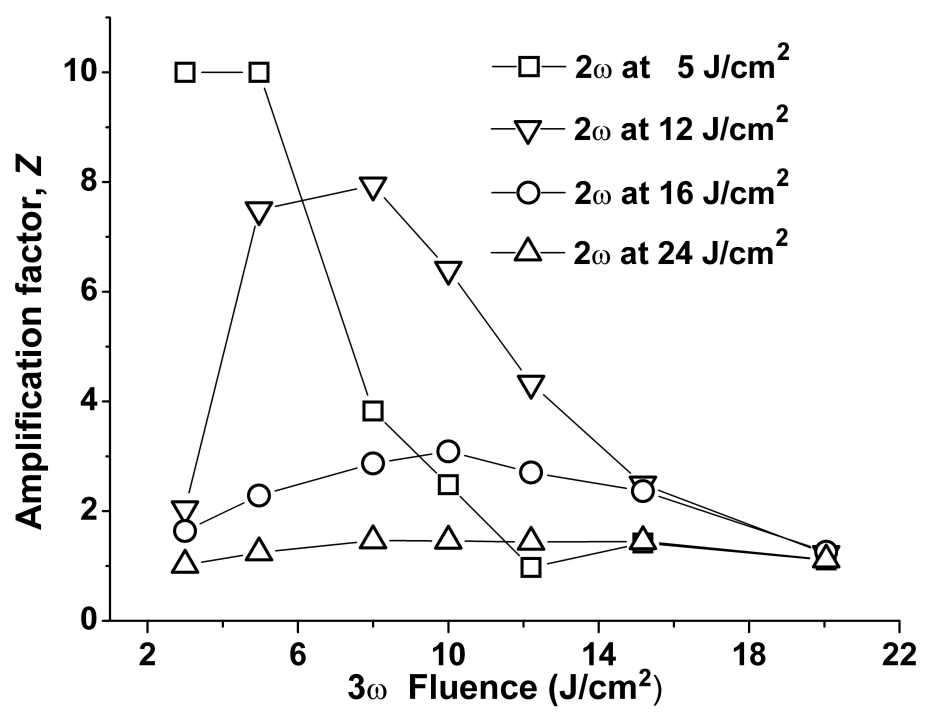

Figure 3. Damage amplification factor for simultaneous $2 \omega$ and $3 \omega$ irradiation.

An important behavior of the data presented in Fig. 2(b) is revealed if we first measure the $3 \omega$ only profile over the entire range of PPDs [as shown in Fig. 4(a)] and then translate each PPD profile $(i)$ at constant $2 \omega$ fluence $\left(\Phi_{2 \omega, i}\right)$ along the $3 \omega$ fluence axis by the amount $\Delta \Phi_{3 \omega, i}$ defined by:

$$
\operatorname{PPD}\left[\Phi_{2 \omega, i}, 0\right]=\operatorname{PPD}\left[0, \Delta \Phi_{3 \omega, i}\right] .
$$


In other words, Eq. 2 is equivalent to translating each PPD profile in Fig. 4(a) such that the first point overlaps the corresponding PPD value on the $3 \omega$ only profile, within experimental errors. After applying this method to all curves, we observe the correlation of PPD profiles from simultaneous exposure to a single profile, as illustrated in Fig. 4(b) by the best fit with a coefficient of determination $\left(\mathrm{R}^{2}\right)$ of 0.96 or greater.
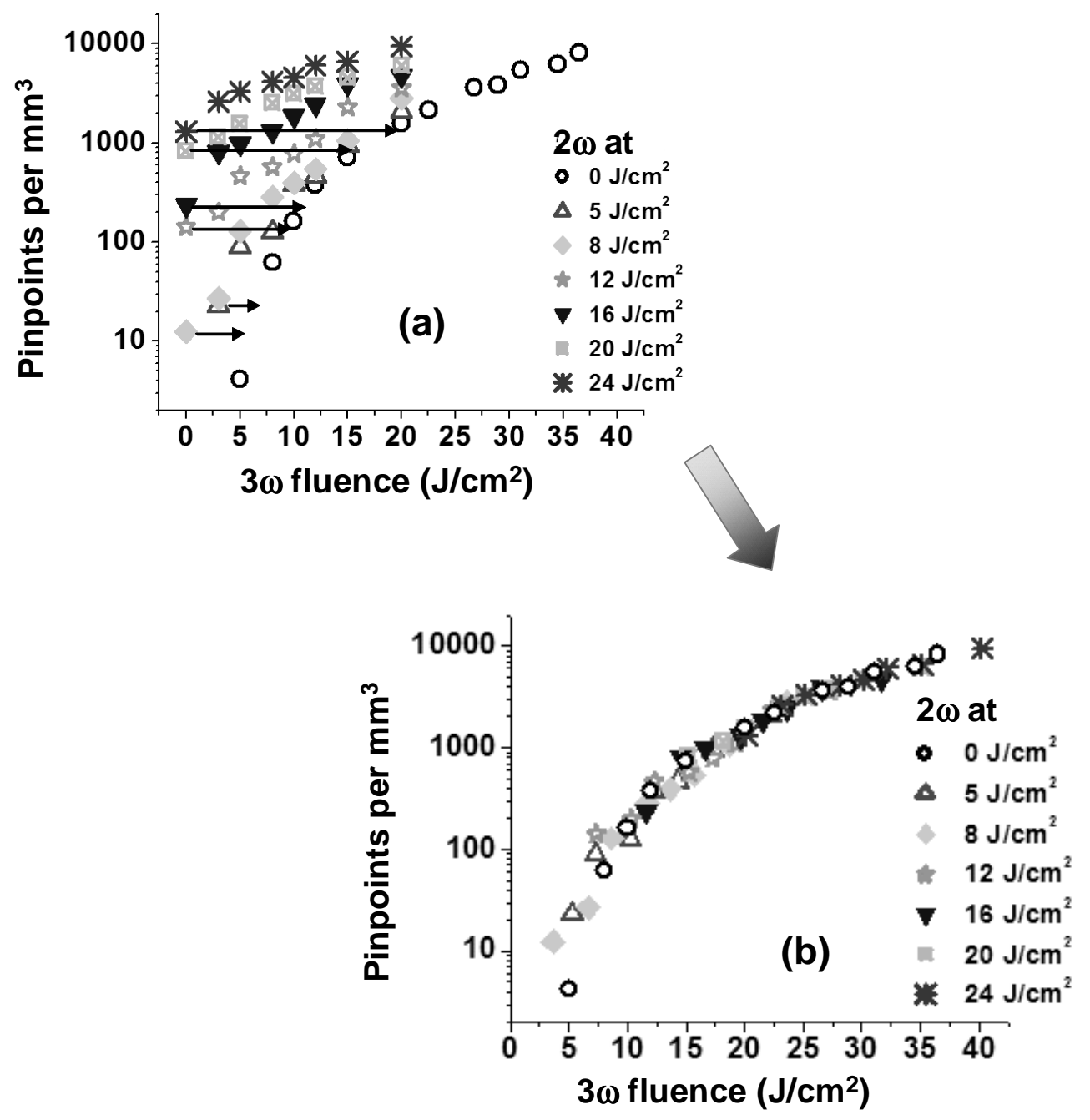

Figure 4. Correlation of all PPD profiles from simultaneous exposure [at constant $2 \omega$ fluence, (a)] to the single profile from $3 \omega$ only [open circles data,(b)], after application of Eq. 2 and best fit for all curves in (a).

Several observations can be inferred from Fig. 4(b): i) all PPD profiles obtained under $2 \omega$ and $3 \omega$ irradiation are part of the unique, $3 \omega$ only profile over different ranges of PPD values and ii) the $2 \omega$ fluence during simultaneous exposure can be replaced by an effective $3 \omega$ fluence which results in the same PPD (see also Eq. 2). We thus conclude that damage initiators involved at $2 \omega$ and $3 \omega$ are the same and initiate damage in the same sequence. In addition, the damage initiation mechanism is the same at both $2 \omega$ and $3 \omega$.

From the correlation of all PPD profiles in Fig. 4(b), Eq. 2 can be re-written in a slightly different form as:

$$
\operatorname{PPD}\left[\Phi_{2 \omega}, \Phi_{3 \omega}\right]=\operatorname{PPD}\left[0, \Phi_{3 \omega}+\Phi_{3 \omega, \mathrm{eff}}\left(\Phi_{2 \omega}\right)\right]
$$

where $\Phi_{3 \omega, \text { eff }}\left(\Phi_{2 \omega}\right)$ is the effective $3 \omega$ fluence which can replace the $2 \omega$ fluence during simultaneous exposure, leading to equivalent damage effects. From Eqs. 2 and 3, we identify $\Phi_{3 \omega, \text { eff }}\left(\Phi_{2 \omega, i}\right)$ as the amount of shift along the fluence axis applied to each PPD profile $(i)$ at constant $\Phi_{2 \omega, i}$ [see Fig. 4(a)] and arrive to a more general definition of the effective $3 \omega$ fluence by setting $\Phi_{3 \omega}=0$ in Eq. 3 :

$$
\operatorname{PPD}\left[\Phi_{2 \omega}\right]=\operatorname{PPD}\left[\Phi_{3 \omega, \text { eff }}\right]
$$

Figure 5 illustrates the effective $3 \omega$ fluence values as a function of $2 \omega$ fluence inferred from the simultaneous exposure profiles shown in Fig. 4(a). 


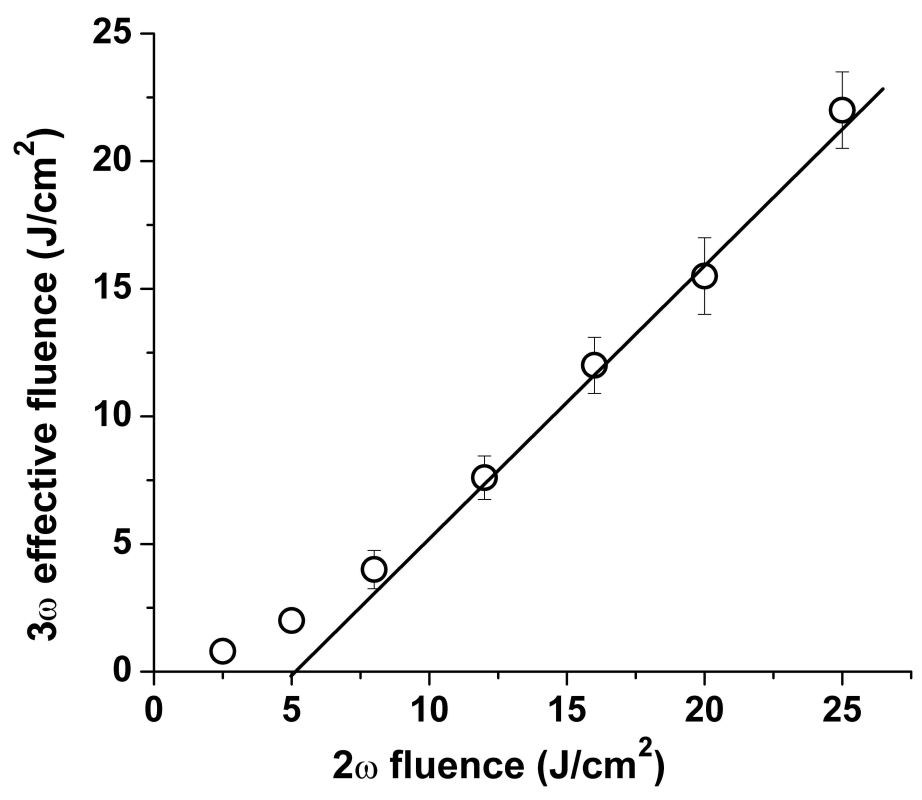

Figure 5. Effective $3 \omega$ fluence as a function of $2 \omega$ fluence which leads to equivalent damage effects under simultaneous $2 \omega$ and $3 \omega$ irradiation (see Fig. 4).

The approach described above for analysis of the data in Fig. 2 can be used to predict the damage performance of DKDP material during frequency conversion without performing the extensive damage testing outlined in this work. Specifically, starting from the single wavelength PPD profiles which are routinely measured for a given sample, Eq. 4 can be used to compute the values of $\Phi_{3 \omega, \text { eff }}$ for arbitrary $2 \omega$ fluences (experimental data is obtained for fluences above the damage threshold of the material, i.e. $\geq 7.5 \mathrm{~J} / \mathrm{cm}^{2}$ ). The procedure is depicted in Fig. 6, illustrating the inverted profiles at $2 \omega$ and $3 \omega$, i.e. damage test fluence as a function of resulting PPD, and line fits to the data (solid curves). The fluences at $2 \omega$ and $3 \omega$ corresponding to the same PPD can then be extracted (see dashed lines in Fig. 6).

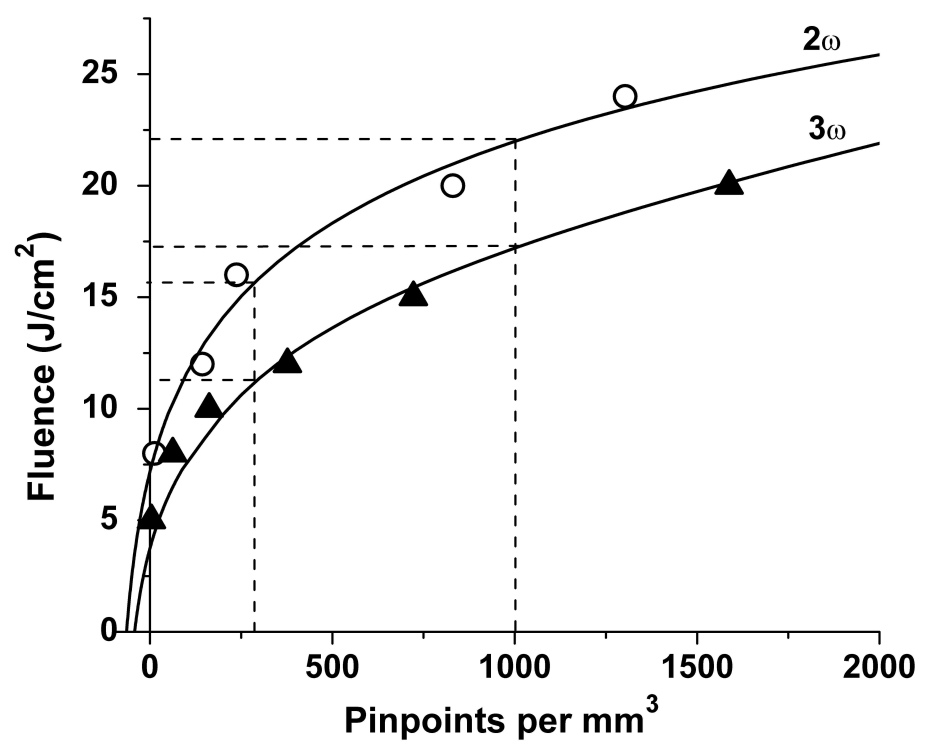

Figure 6. Single wavelength damage profiles at $2 \omega$ and $3 \omega$ in DKDP (damage testing fluence vs. PPD).Equation 4 is used to correlate the damage behaviors between the two frequencies (indicated by dashed lines). The solid lines represent fits to the data.

In order to use Eq. 4 with $2 \omega$ and $3 \omega$ fluence values below the damage threshold, where the material is expected to operate during frequency conversion, the profiles shown in Fig. 6 need to be extended beyond the origin, in the region of negative PPD values. Alternatively, we can use the profile shown in Fig. 5 as a calibration method for arbitrary samples in order to extrapolate the dependence of the effective $3 \omega$ fluence on the $2 \omega$ fluence for values below the damage 
threshold.

As an example, a fluence of $\sim 16 \mathrm{~J} / \mathrm{cm}^{2}$ at $2 \omega$ corresponds to $\sim 12 \mathrm{~J} / \mathrm{cm}^{2}$ at effective $3 \omega$ fluence, both resulting in $\sim 300$ pinpoints per $\mathrm{mm}^{3}$. Similarly, $\sim 22 \mathrm{~J} / \mathrm{cm}^{2}$ at $2 \omega$ is equivalent to $\sim 17.5 \mathrm{~J} / \mathrm{cm}^{2}$ at effective $3 \omega$ (corresponding to $\sim 1000$ pinpoints per $\mathrm{mm}^{3}$ ). Finally, the PPD under simultaneous exposure to $16 \mathrm{~J} / \mathrm{cm}^{2}$ at $2 \omega$ and $5 \mathrm{~J} / \mathrm{cm}^{2}$ at $3 \omega$ can be predicted to be $\sim 900$ pinpoints per $\mathrm{mm}^{3}$, as confirmed from our measurements in Fig. 2(b):

$$
\operatorname{PPD}\left[16 \mathrm{~J} / \mathrm{cm}_{2 \omega}^{2}, 5 \mathrm{~J} / \mathrm{cm}_{3 \omega}^{2}\right]=\operatorname{PPD}\left[0,12 \mathrm{~J} / \mathrm{cm}_{3 \omega, \text { eff }}^{2}+5 \mathrm{~J} / \mathrm{cm}_{3 \omega}^{2}\right]=\operatorname{PPD}\left[17 \mathrm{~J} / \mathrm{cm}_{3 \omega}^{2}\right] \text {. }
$$

\section{SUMMARY}

We have investigated the damage behavior of a nonlinear material at conditions that approximate those during its normal operation. Our results provide insight into the expected behaviors as well as an opportunity to reveal key fundamental behaviors involved in the damage initiation process. Experimental data demonstrate that simultaneous presence of $2 \omega$ and $3 \omega$ light leads to enhanced damage effects which need to be accounted for during harmonic generation. The defect structures responsible for damage at $2 \omega$ and $3 \omega$ are substantially the same and initiate damage in the same sequence (Ref. 8 provides additional evidence to support these statements). In addition, the damage mechanism is the same. This work also provides an empirical method to predict damage performance of DKDP material under simultaneous exposure to $2 \omega$ and $3 \omega$ light.

\section{ACKNOWLEDGMENTS}

This work was performed under the auspices of the U.S. Department of Energy by University of California, Lawrence Livermore National Laboratory under contract no. W-7405-Eng-48.

\section{REFERENCES}

1. J. J. De Yoreo, A. K. Burnham, and P. K. Whitman, "Developing $\mathrm{KH}_{2} \mathrm{PO}_{4}$ and $\mathrm{KD}_{2} \mathrm{PO}_{4}$ crystals for the world's most powerful laser," Int. Mater. Rev. 47, pp. 113-152, 2002.

2. N. Zaitseva, J. Atherton, R. Rozsa, L. Carman, I. Smolsky, M. Runkel, R. Ryon, and L. James, "Design and benefits of continuous filtration in rapid growth of large KDP and DKDP crystals," J. Cryst. Growth 197, pp. 911-920, 1999.

3. A. K. Burnham, M. Runkel, M. D. Feit, A. M. Rubenchik, R. L. Floyd, T. A. Land, W. J. Siekhaus, and R. A. Hawley-Fedder, "Laser-induced damage in deuterated potassium dihydrogen phosphate," Appl. Opt. 42, pp. 5483$5495,2003$.

4. R. M. Wood, Laser-Induced Damage of Optical Materials, Institute of Physics, Bristol, 2003.

5. H. Yoshida, T. Jitsuno, H. Fujita, M. Nakatsuka, M. Yoshimura, T. Sasaki, and K. Yoshida, "Investigation of bulk laser damage in KDP crystal as a function of laser irradiation direction, polarization and wavelength," Appl. Phys. B 70, pp. 195-201, 2000.

6. C. W. Carr, M. D. Feit, A. M. Rubenchik, M. Spaeth, and J. B. Trenholme, "Summary of recent experiments in bulk damage initiation in KDP and their interpretation," this SPIE Proceedings, Paper No. 5991-28.

7. P. DeMange, R. A. Negres, C. W. Carr, H. B. Radousky, and S. G. Demos, "A new damage testing system for detailed evaluation of damage behavior of bulk KDP and DKDP," in Laser-Induced Damage in Optical Materials: 2004, G. J. Exarhos, A. H. Guenther, N. Kaiser, K. L. Lewis, M. J. Soileau, and C. J. Stolz, eds., Proc. SPIE 5647, pp. 343-353, 2005.

8. P. DeMange, R. A. Negres, H. B. Radousky, and S. G. Demos, "Different precursor populations revealed by microscopic studies of bulk damage in KDP and DKDP crystals," this SPIE Proceedings, Paper No. 5991-87. 